\title{
Creep or relaxation master curves calculated from experimental dynamic viscoelastic function
}

\author{
Rui Miranda Guedes, António Torres Marques \\ Departamento de Engenharia Mecânica e Gestão Industrial da Faculdade de Engenharia, \\ Universidade do Porto, Rua dos Bragas, 4099 Porto Codex, Portugal
}

\section{Albert Cardon}

Vrije Universiteit Brussel (V.U.B.) Dept. of Mechanics of Materials and Construction (MEMC), Pleinlaan 2, B-1050 Brussels, Belgium

\begin{abstract}
The problem treated consists in obtaining the unknown creep or relaxation master curves from experimental dynamic viscoelastic functions obtained on a DMTA analyser. In the present work, a general discrete model, the generalised Kelvin model or the generalised Maxwell model, is used to fit the complex compliance or the complex modulus respectively. A linear regression technique is applied to the measured data in an iterative calculation, varying the relaxation or the retardation times to obtain the minimum of the relative error. For a physical meaning, the admissible solutions do not include negative relaxation or retardation times. The method may be used to obtain an approximation of the discrete relaxation or retardation spectra from dynamic experimental data. The error within this procedure is discussed.
\end{abstract}




\section{Introduction}

When characterising the mechanical properties of polymeric materials on a DMTA analyser, we obtain the complex modulus $E^{*}$ as a function of angular frequency $w$. In order to characterise the viscoelastic material behaviour in a very broad range of frequencies (or times), it is necessary to combine measurements at several temperatures applying the time-temperature superposition principle (TTSP). According to this principle, a given property measured for short times at higher temperature must be the same as that obtained for longer times at lower temperature, except that the curves are shifted parallel to the horizontal axis, matching to a master curve. Povolo [1] demonstrated that if the curves that describe the time or frequency dependence of the mechanical properties at different temperatures obey the time-temperature superposition principle, then the corresponding individual distribution functions can also be matched to form a master spectrum. These superposition properties of spectra will be used in this paper.

The discussions are based on a linear viscoelastic material behaviour having a positive relaxation or retardation spectrum. The exact formal relationships between the various viscoelastic functions are conveniently expressed using Fourier or Laplace integral transforms. The complex relaxation modulus is given by

$$
G^{*}(\omega)=G_{0}+i \omega \int_{0}^{\infty} G(\tau) e^{-i \omega \tau} d \tau
$$


where $G^{*}(\omega)=\left(G_{0}+G_{1}\right)+i G_{2}$ and $\lim _{w \rightarrow \infty} G^{*}(w)=G_{0}$. Equation 1 contains a Fourier transform. Conversely the relaxation modulus is given by

$$
\frac{d G(t)}{d t}=F^{-1}\left[G^{*}(w)-G_{0}\right]
$$

where $F^{-1}$ represents the inverse Fourier transform.

Then

$$
G(t)=G_{0}+\int_{0}^{t} F^{-1}\left[G^{*}(w)-G_{0}\right] d \tau
$$

The approximation of the kernels requires the computation of various derivatives of the experimental data, and this limits the order of the approximation that may be used. In practice the calculation of the integral transforms, which have to be evaluated over an infinite time or frequency regime extending from zero to infinity, often fails due to the lack of information about the measured function.

Much work was done to develop simple numeric formulae for the interconversions that do not involve any integration of the function measured. If both dynamic components are known, the transient function can be calculated by the method of Ninomiya and Ferry [2]. Schwarzl [3] published a set of error-bound approximations for the interconvertion of viscoelastic material functions, which are based on approximation of the kernel functions in the spectral representation of the mechanical properties.

More recently Gibson [4] proposed the use of frequency domain vibration test data to obtain time-dependent creep and relaxation properties of polymer 
composite materials. The Fast Fourier Transform (FFT) algorithm and numerical integration were employed to solve Equation 3.

P. Mailler, Bigaud and Hamelin [5] used a fractional model, the Zener biparabolic model, to represent the master curves of the dynamic modulus. The relaxation modulus was obtained with approximate inversion methods.

The spectra provide useful information about the polymer properties, provide that viscoelastic functions are measured over many decades. A spectrum is a material function, independent of the way of excitation. Once the spectrum and viscoelastic constants are known, it is possible, in theory, to generate the response to any desired type of excitation. The spectrum is not accessible by direct experiment [6], but it is often possible to calculate it exactly from a mathematical model of a response. The determination of spectra involves the inversion of a Fredholm integral equation of the first kind. Because this is an ill-posed problem [7], for each set of experimental data a large number of different spectra can be found, each of them describing the data with the same accuracy.

To overcome these difficulties, several authors proposed special methods to determine the spectra from an experimentally obtained function. Most of the methods published in the 1950s and 1960s were set up to yield continuous spectra. Extensive discussions of the means available to obtain approximations to the continuous spectra can be found in the texts by Ferry [2], and by Tschoegl [8]. Due to the easy availability of sufficiently powerful computers, the last several years have seen an increase in interest in methods for computing spectral distributions. Thigpen [7] used a numerical technique to invert creep 
compliance data for retardation spectra and to determine dynamic viscoelastic functions from spectra. It uses constrained regularization, in which the solution is constrained to be non negative. This method was shown to give accurate results, even in the presence of noise. Yong-Sok et. al. [9] characterised the viscoelastic dynamic properties of carbon fibre reinforced epoxy, using the Zener biparabolic model to fit the dynamic master curves. The mathematical expression of the relaxation time spectra of the model can then be readily obtained. Recently several recursive computer algorithms were proposed. The most convenient algorithm for determining discrete spectra, equally spaced on the logt axis, is probably the algorithm of Emri and Tschoegl [6], [10], [11]. This algorithm scans the data trough "windows" over a span of two logarithmic decades. This "windowing" circumvents the ill-posed problem inherent in solving the Fredholm integral equations of the first kind, when an attempt is made to use the entire data. More recently Kaschta and Schwarzl [12], [13] proposed a new method for the calculation of discrete retardation spectra from creep or recovery data. A linear regression technique was applied to the measured data in the iterative calculation of a spectrum with logarithmic equidistant spacing of retardation times. Because the number of retardation times was limited, the ill-posed problem was avoided.

In the following, a new method is presented and applied to simulated dynamic data to obtain the creep or relaxation master curves. Comparisons with exact responses are then used to illustrate the applicability of the method. 


\section{Theoretical Basis}

The retardation time spectrum and the relaxation time spectrum are mathematical descriptions of the materials macroscopic behaviour and do not have a simple interpretation in molecular terms. The retardation time spectrum is a quantitative description of creep and forms a link with the original mathematical descriptions of linear viscoelastic theory with the Boltzmann integrals. The creep compliance $J(t)$ can be calculated from the non-negative retardation time spectrum $L(\tau)$ by the equation

$$
J(t)=J_{0}+\int_{-\infty}^{+\infty} L(\tau)\left(1-e^{-\frac{t}{\tau}}\right) d \ln \tau
$$

where $J_{0}$ is the instantaneous elastic compliance. Similar relationships hold between the real and imaginary parts of the complex compliance $J^{\prime}, J^{\prime \prime}$ and the retardation time spectrum $L(\tau)$. The dynamic behaviour under prescribed sinusoidal stress can be calculated as

$$
\begin{gathered}
J^{\prime}(\omega)=J_{0}+\int_{-\infty}^{+\infty} L(\tau) \frac{1}{1+\omega^{2} \tau^{2}} d \ln \tau \\
J^{\prime \prime}(\omega)=\int_{-\infty}^{+\infty} L(\tau) \frac{\omega \tau}{1+\omega^{2} \tau^{2}} d \ln \tau
\end{gathered}
$$

The experimental functions can be described with the same precision by a discrete retardation spectrum. The retardation functions given by equations 4 through 6 can be expressed in terms of discrete spectral distributions as

$$
J(t)=J_{0}+\sum_{k=1}^{N} J_{k}\left(1-e^{-\frac{t}{\tau_{k}}}\right)
$$




$$
\begin{gathered}
J^{\prime}(\omega)=J_{0}+\sum_{k=1}^{N} J_{k} \frac{1}{1+\omega^{2} \tau_{k}^{2}} \\
J^{\prime \prime}(\omega)=\sum_{k=1}^{N} J_{k} \frac{\omega \tau_{k}}{1+\omega^{2} \tau_{k}^{2}}
\end{gathered}
$$

where the sets $\left\{J_{k}, \tau_{k}\right\}$, for $k=1,2, \ldots, N$, represent the discrete distribution of retardation times. For physical reasons the retardation spectrum and all the constants $\left\{J_{k}, \tau_{k}\right\}$ should be non-negative. The equations involving the logarithmic relaxation spectrum, $H(\tau)$, or the discrete relaxation spectra, $\left\{G_{k}, \bar{\tau}_{k}\right\}$, are quite similar.

\section{Algorithm}

We have a set of $\mathrm{M}$ pairs of measured data points $\left(\varphi_{1}^{\prime}, \varphi_{1}^{\prime \prime}\right),\left(\varphi_{2}^{\prime}, \varphi_{2}^{\prime \prime}\right), \ldots$, $\left(\varphi_{M}^{\prime}, \varphi_{M}^{\prime \prime}\right)$ of a complex function $\varphi^{*}$, in our case the complex compliance, at the observation points of frequencies $f_{1}, f_{2}, \ldots, f_{M}$

$$
\begin{gathered}
\varphi^{\prime}\left(f_{m}\right) \equiv J^{\prime}\left(\omega_{m}\right)=J_{0}+\sum_{k=1}^{N} J_{k} \frac{1}{1+\omega_{m}^{2} \tau_{k}^{2}} \\
\varphi^{\prime \prime}\left(f_{m}\right) \equiv J^{\prime \prime}\left(\omega_{m}\right)=\sum_{k=1}^{N} J_{k} \frac{\omega_{m} \tau_{k}}{1+\omega_{m}^{2} \tau_{k}^{2}}
\end{gathered}
$$

for $m=1,2, \ldots, M$ and with $\omega_{m}=2 \pi f_{m}$.

The problem to be solved is how to determine the $2 N+1$ unknown parameters which fit the experimental response best. The number of retardation times, $N$, is an open choice, in this case it was chosen to be smaller than the number of measuring points, $2 M$. 
The experimental frequency window we are interested is small enough to avoid the ill-posed problem. In most cases the dynamic data are measured at frequencies in the range 0.01 to $30 \mathrm{~Hz}$ or in the range 0.1 to $50 \mathrm{~Hz}$.

In theory both Equations 10 and 11, independently, should give the same results represented by the sets $\left\{J_{k}, \tau_{k}\right\}_{k=1,2, \ldots, N}$. However, since $J^{\prime}(\omega)$ and $J^{\prime \prime}(\omega)$ contain different experimental errors, they cannot give exactly the same solutions. Due to these unavoidable differences we decided to combine both equations to determine the unknown parameters.

The first method published to solve this kind of problems was the collocation method, which was suggested by Schapery [14]. This method linearizes the problem by prescribing those parameters that make the problem non-linear, in this case the $\left\{\tau_{k}\right\}_{k=1,2, \ldots, N}$ parameters. The remaining parameters, $J_{0}$ and $\left\{J_{k}\right\}_{k=1,2, \ldots, N}$, are determined by a linear system of equations, where the number of data points is the same as the number of unknown parameters. Therefore, all data points used are exactly reproduced by the theoretical curve. The main problem of collocation method is that it only works successfully if the spacing of the collocation points is neither too large nor too close, otherwise negative parameters are obtained. The result of the collocation method naturally depends on the choice of the number of data points, their location and the prescribed parameters $\left\{\tau_{k}\right\}_{k=1,2, \ldots, N}$.

The method proposed by Kaschta and Schwarzl [12] is an iterative multidata method. As they refer, the multidata method uses all $M$ data points available to determine the $N+1$ parameters, $J_{0}$ and $\left\{J_{k}\right\}_{k=1,2, \ldots, N}$. The system is overdetermined and the problem is solved in the least square sense. 
The present algorithm does not expect any type of distribution of the measuring frequencies, so they could be logarithmic equidistant or distributed in an irregular manner on the logarithmic frequency axis. The retardation times, $\left\{\tau_{k}\right\}_{k=1,2, \ldots, N}$, are prescribed as logarithmic equidistant:

$$
t_{k}=t_{1} \cdot a^{k-1}, k=1,2, \ldots, N
$$

In the measurements taken, it is the relative experimental error, which is approximately independent of the frequency, that takes into account the order of the magnitude of the compliance measured. This effectively equalises the influence of the errors associated with all data points within the frequency window. Therefore we adopted the following parameter for the quality of the description of the data:

$$
\Psi=\sum_{i=1}^{M}\left(\frac{\varphi^{\prime}\left(f_{i}\right)}{\varphi_{i}^{\prime}}-1\right)^{2}+\sum_{i=1}^{M}\left(\frac{\varphi^{\prime \prime}\left(f_{i}\right)}{\varphi_{i}^{\prime \prime}}-1\right)^{2}
$$

where $\left\{\varphi_{i}^{\prime}, \varphi^{\prime \prime}\right\}_{i=1,2, \ldots, M}$ represent the sets of measured dynamic compliance data. Minimising the sum of squares of relative errors $\Psi$, concerning the set of parameters to be determined, $J_{0}$ and $\left\{J_{k}\right\}_{k=1,2, \ldots, N}$, yields a set of linear equations for the calculations of those parameters as

$$
\frac{\partial \Psi}{\partial J_{0}}=0 \wedge \frac{\partial \Psi}{\partial J_{k}}=0(k=1,2, \ldots, N)
$$

This leads to the following linear system of equations written in matrix form as 
where

$$
A^{T} \cdot A \cdot\left\{\begin{array}{c}
J_{0} \\
J_{1} \\
\vdots \\
J_{N}
\end{array}\right\}=A^{T} \cdot\left\{\begin{array}{c}
1 \\
1 \\
\vdots \\
1
\end{array}\right\}
$$

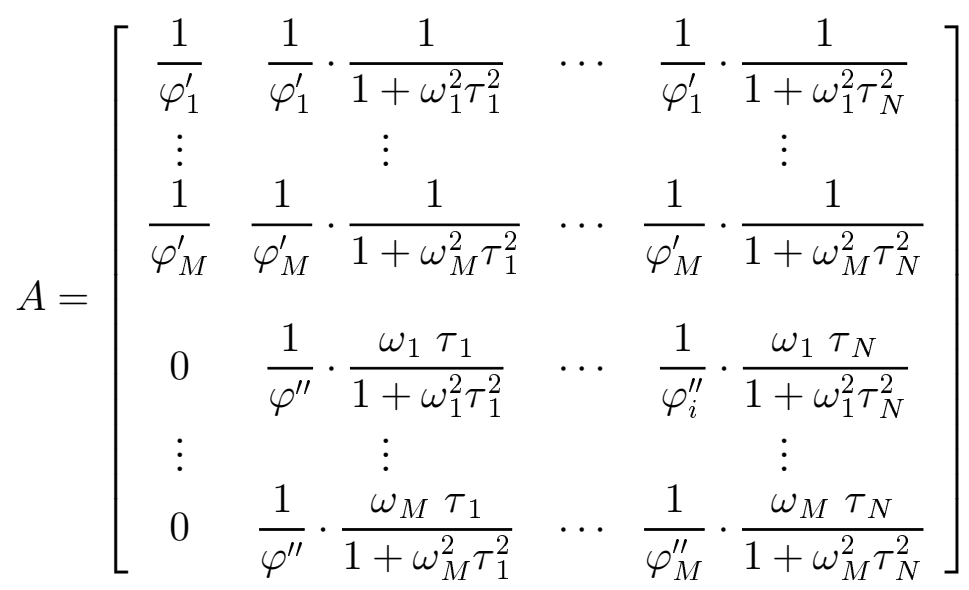

In our method, after fixing the value of the parameter $a$ in Equation 10, we start with the choice for the first retardation time

$$
\tau_{1}=b
$$

with $b=10^{-2} / f_{\max }$. In order to limit the number of modes and to ensure that the reversed retardation time window $\left[1 / \tau_{N}, 1 / \tau_{1}\right]$ does not exceed significantly the frequency window $\left[f_{\min }, f_{\max }\right]$ of the experimental data, the number of retardation times should respect the following condition.

$$
N \leq \text { Integer }\left[\frac{\log \left(f_{\max } / f_{\min }\right)}{\log a}\right]+2
$$

The set of parameters is calculated using the equation (16) under the condition expressed in Equation 18. After, the b value is substituted by a slightly larger value as 


$$
b \rightarrow b+\epsilon_{b}
$$

with a small $\epsilon_{b}$ value and the process is repeated, until a set of positive parameters $J_{0}$ and $\left\{J_{k}\right\}_{k=1,2, \ldots, N}$, which minimises the value of $\Psi$, is found. These set are used as the optimum description of the dynamic data. As Kaschta and Schwarzl [12] reported, there is a window, on the time axis for $\tau_{1}$ for each value of $a$, which yields only positive retardation parameters.

The method is illustrated in Figure 1, where the course minimum of the of the sum of squares of relative errors, $\Psi$, and the correspondent first retardation time are shown as a function of the logarithmic distance, a, between the retardation times to calculate dynamic compliances from the simulated curves presented in Table I (derived from the case defined later in Equation 20). As one can see in Figure 1, the optimum solution depends on $a$ and $b$ constants. In this case we concluded that $b=2.5$ gives the best results.

\section{Tests}

We tested the algorithm for a wave-like spectrum previously studied by Swanson and reported by Thigpen [7] in his investigation. The logarithmic retardation spectra adopted was

$$
L(\tau)=\left[1-e^{-(10 \cdot \tau-1)}\right] \cdot e^{-\tau} \cdot H(\tau-1)
$$

with $J_{0}=0.147 \mathrm{GPa}^{-1}$ being $\mathrm{t}$ in seconds. This spectrum simulates the quartz monzonite spectra at $1003^{\circ} \mathrm{K}$ and $180 \mathrm{MPa}$ confining pressure. The dynamic compliances, $J^{\prime}$ and $J^{\prime \prime}$, calculated by numerical integration of Equations 5 
and 6, for the spectra given by Equation 20, are presented in Table I. The frequencies were chosen to be coincident with the frequencies used for the measurements on a DMTA analyser. Only these points, $M=7$, were used in order to determine the optimum solution of Equations 8 and 9 represented by $J_{0}$ and the sets $\left\{J_{k}, \tau_{k}\right\}_{k=1,2, \ldots, N}$.

Figures 2 and 3 show the discrete spectra and creep compliance as calculated from the dynamic data, presented in Table I, with $a=2.5$ and $N=5$, together with the exact functions.

Both normalised discrete spectra, given by $\left\{\tau_{k}, J_{k} / \ln (a)\right\}$, and the exact spectrum, given by Equation 20, coincide very well on the scale of Figure 2. In Figure 3 one cannot see significant differences between the calculated values and the exact ones for the creep compliance, originating relative errors lower than $0.5 \%$.

The calculations presented before have been performed with simulated errorfree data. To test the algorithm with noisy data, like those which are extracted from the experiments, we imposed some random noise on the dynamic data that is shown in Table I. This was done by multiplying the exact values with the $(1+\partial \cdot \operatorname{rand}(i))$ factor, where $\partial$ is a measure of the relative experimental error and $\operatorname{rand}(i)$ is a random number between -1 and +1 .

In Figure 4, the course minimum of the of the sum of squares of relative errors, $\Psi$, and the correspondent first retardation time are shown as function of the logarithmic distance, $a$, between the retardation times for the calculation of dynamic compliances with $10 \%$ noise. As can be seen in Figure 4, the optimum solution for the $\pm 10 \%$ noise data is "less" dependent on $a$ and presents an 
enlarged window for positive parameters in comparison with the error-free data case.

In Figures 5 and 6 the resulting discrete spectra and creep compliance calculated, respectively, from $\pm 10 \%$ noise $(\partial=0.1)$ dynamic data with $a=2.5$ and $N=5$, is plotted together with the exact functions. The superimposed noise influences the course of the spectra in the peak region. For the calculated creep compliance we found relative errors lower than $9 \%$. In this case, the noisy data did not cause severe variations of the output results, showing the good efficiency of the algorithm.

For more general cases, we simulated tests conducted on a DMTA test machine dynamic analyser. Frequencies of 0.01, 0.02, 0.03, 0.10, 1.0, 10.0 and $30.0 \mathrm{~Hz}$ were used at 9 different temperatures, $\mathrm{T}$, from 30 up to $110^{\circ} \mathrm{C}$. The master retardation spectrum was used to generate the dynamic viscoelastic functions.

Two shapes for the logarithmic retardation spectrum were selected. The first has a multi-step-shape

$$
L_{m s}(\tau)= \begin{cases}0 & \text { for } \quad \tau<10^{-3} \bigwedge \tau \geq 10^{15} \\ 10^{-3} & \text { for } 10^{-3} \leq \tau<10^{2} \\ 10^{-1} & \text { for } 10^{2} \leq \tau<10^{7} \\ 10^{0} & \text { for } 10^{7} \leq \tau<10^{15}\end{cases}
$$

The other spectrum is derived from the compliance function of an epoxy resin, which is based on the Cole-Cole function [8], using the second order approximation formula of Schwarzl and Staverman [13] 


$$
L_{c c}(\tau)=\frac{9.433 \times 10^{7} \cdot\left[1.485+9.956 \times 10^{5} \cdot\left(\frac{1}{\tau}\right)^{0.485}\right] \cdot\left(\frac{1}{\tau}\right)^{0.485}}{\left[1+1.933 \times 10^{6} \cdot\left(\frac{1}{\tau}\right)^{0.485}\right]}
$$

$L(\tau)$ is calculated in $\mathrm{GPa}^{-1}$ and $\tau$ in hours, with the additional parameter $J_{0}=0.147 \mathrm{GPa}^{-1}$. These spectra have been chosen to describe approximately the master curves of amorphous uncrosslinked polymers, Equation 21 and epoxy resins, Equation 22.

According to TTSP, the viscoelastic response at a higher temperature is equivalent to that of a lower temperature but for a longer time duration. Therefore, by translation of the small curves measured at different temperatures along a logarithmic time axis, a large master curve can then be constructed at a reference temperature $T_{0}$. The curve of each temperature is shifted an amount of $\log \left(a_{T}\right)$. For both simulated cases, the $a_{T}-T$ relation is described by an Arrhenius equation as

$$
\log _{10} a_{T}=\frac{\Delta H}{2.303 \cdot R}\left(\frac{1}{T}-\frac{1}{T_{0}}\right)
$$

where $\Delta H$ is the activation energy for the relaxation process and

$$
\left\{\begin{array}{lcl}
T<333.15^{\circ} \mathrm{K}: & \Delta H=60.0 \mathrm{Kcal} / \mathrm{mol} ; & T_{0}=303.15^{\circ} \mathrm{K} \\
T \geq 333.15^{\circ} \mathrm{K}: & \Delta H=120.0 \mathrm{Kcal} / \mathrm{mol} ; & T_{0}=317.44^{\circ} \mathrm{K}
\end{array}\right.
$$

The numerical procedure is as follows. At each temperature, the dynamic data are transformed by the algorithm into time domain. Then, the data are shifted for each temperature to a reference temperature $T_{0}\left(30^{\circ} \mathrm{C}\right.$ in this case $)$ by using the time-temperature superposition principle (TTSP) to build up the 
master creep compliance curve. The curve of each temperature $\mathrm{Tj}$ is shifted over an amount of $\log \left(a_{T}\right)$ as

$$
J_{T_{0}}[\log (t)]=J_{T_{j}}\left[\log (t)-\log \left(a_{T}\right)\right]
$$

The parameter $a_{T}$ is the temperature-time scale factor. Povolo [1] demonstrated that if the curves that describe the time or frequency dependence of the mechanical properties at different temperatures obey the TTSP, then, the corresponding individual distribution functions can also be matched to form a master spectrum, and, vice versa. So for each point of the discrete spectrum at temperature $T_{j}$, we can then apply the following transformation to form the master retardation spectrum at the reference temperature $T_{0}$

$$
\left\{\tau_{i}, J_{i}\right\}_{T_{j}} \Rightarrow\left\{\tau_{i} / a_{T}, J_{i}\right\}_{T_{0}}
$$

For the simulated dynamic data, consequently error-free data, we concluded that $a=10$ and $N=5$ lead to the best results and therefore were adopted these values for all subsequent calculations.

In Figure 7 the calculated spectra for the multi-step spectrum are given, and in Figure 8 the calculated compliance (approx.) is plotted against the exact function (exact) along with the relative error. Regions of constant value in the spectrum are reproduced very well and the jumps in the multi-step spectrum are reasonably reproduced. As shown in Figure 8 the approximation of the creep compliance shows a relative error lower than $4.0 \%$.

In Figure 9 the calculated values for the spectrum of Equation 22 are shown, and in Figure 10 the calculated creep compliance (approx.) is plotted against 
the exact function (exact) with the relative error. The discrete spectra and the exact spectrum coincide very well on the logarithmic scale of Figure 9. As shown in Figure 10 the approximation of the creep compliance exhibits a relative error lower than $4.0 \%$.

We will demonstrate that the method still works with noisy data for these cases. As in the first test we impose some random noise on the dynamic data by multiplying the exact values by a factor of $(1+\partial \cdot \operatorname{rand}(i))$.

In Figures 11 and 12 the calculated creep compliance (approx.) is plotted against the exact function (exact) along with the relative error for both cases. It can easily be seen that the calculated curves maintain the shape of the exact curves but are shifted along the time axis. This shifting is the result of cumulative errors in calculation of the temperature-time scale factors due to the random noise. The relative errors depend on the deviation and on the curve's slope and therefore for the same amount of deviation, the relative error increase with the increase of the curve's slope. As for the activation energy of the Arrhenius equation, the present method calculates those parameters with a relative error lower than $7.5 \%$.

\section{Conclusions}

We have presented an easy to use algorithm in order to construct the master creep compliance curves from the experimental dynamic viscoelastic functions obtained on a DMTA analyser. The method can also be used to obtain an approximation of the discrete retardation spectra. 
From error-free data we found that the calculated master creep compliance curve nearly coincides with the exact one. As for the retardation spectrum, we conclude that for regions where the retardation spectrum is either constant or obeys a power law behaviour these are reproduced quite well by the algorithm. Regions of steep changes in the spectrum are still reasonably reproduced, although it tends to scatter near high jumps in the logarithmic scale. Noisy data do not affect significantly the performance of the algorithm here presented, since it acts as a smoothing tool.

\section{Acknowledgements}

The financial support of this work by the JNICT (Junta Nacional de Investigação Científica e Tecnológica) under the Grant BD 706 is gratefully acknowledged.

\section{References}

1. Povoldo, F., Elida B. Hermida, Journal of Materials Science, 25, 4036-4041 (1990).

2. Ferry, John D., Viscoelastic Properties of Polymers, John Wiley \& Sons (1980), pp. 90 .

3. Schwarzl, F. R., Rheol Acta , 14, 581-590 (1975).

4. Gibson, R. F. , S. J. Hwang, C. H. Sheppard, Journal of Composite Materials, 24, (1990). 
5. Mailer, P., Bigaud, D. \& Hamelin, P., "Durability Evaluation on Soft Membrane Composites Under Combined Loading (mechanical and environmental) “, in Progress in Durability Analysis of Composite Systems, Cardon, Fukuda \& Reifsnider(eds.), Balkema, Rotterdam (1996), pp. 195202.

6. $\quad$ Emri, I. \& N. W. Tschoegl, Rheol Acta, 32, 311-321 (1993).

7. Thigpen, L., G. W. Hedstrom, B. P.Bonner, Journal of Applied Mechanics, 50, 361-366 (1983).

8. Tschoegl, N. W., "The phenomenological theory of linear viscoelastic behaviour“, Springer-Verlag (1989).

9. Yong-Sok O., C. Pilot \& C. Jordan, "Modelization of Viscoelastic Behavior of Carbon/Epoxy Unidirectional Composites“, in: Proceeding of the European Mechanics Colloquium 182 (1984), pp. 73-83.

10. Emri, I. \& N. W. Tschoegl, Rheol Acta, 32, 322-327 (1993).

11. Emri, I. \& N. W. Tschoegl, Rheol Acta, 33, 60-70 (1994).

12. Kaschta, J. \& F. R. Schwarzl, Rheol Acta, 33, 517-529 (1994).

13. Kaschta, J. \& F. R. Schwarzl, Rheol Acta, 33, 530-541 (1994).

14. Schapery, R. A., "Approximation methods of transform inversion for viscoelastic stress analysis", in: Proceedings of the 4th U.S. National 
Congress of Applied Mechanics, ASME, 2 (1962), pp. 1075-1085. 
Table I. Dynamic curves for the spectra given by Equation 20 .

\begin{tabular}{|l|l|l|}
\hline$f(H z)$ & $J^{\prime}\left(G P a^{-1}\right)$ & $J^{\prime \prime}\left(G P a^{-1}\right)$ \\
\hline 0.010 & 1.460554 & $5.1210403 * 10^{-02}$ \\
\hline 0.020 & 1.450053 & $9.9951141 * 10^{-02}$ \\
\hline 0.030 & 1.434514 & 0.1449075 \\
\hline 0.100 & 1.275557 & 0.3573597 \\
\hline 1.000 & 0.3862667 & 0.4179713 \\
\hline 10.00 & 0.1509112 & $5.9170481 * 10^{-02}$ \\
\hline 30.00 & 0.1474377 & $1.9819962 * 10^{-02}$ \\
\hline
\end{tabular}




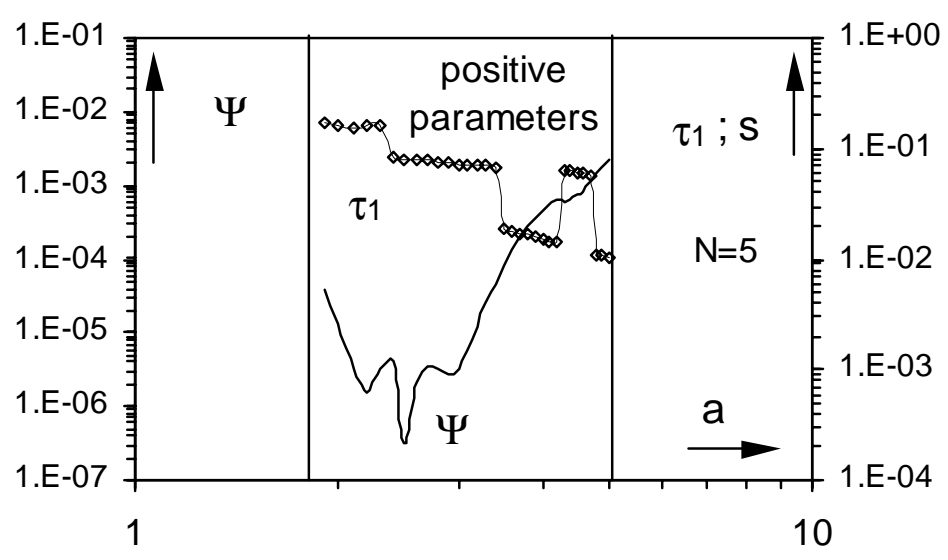

Figure 1: Minimum of the sum of relative errors, $\Psi$, and the corespondent retardation time, $\tau_{1}$, for the calculation of the dynamic compliances, as functions of the logarithmic distance, $a$, between the retardation times.

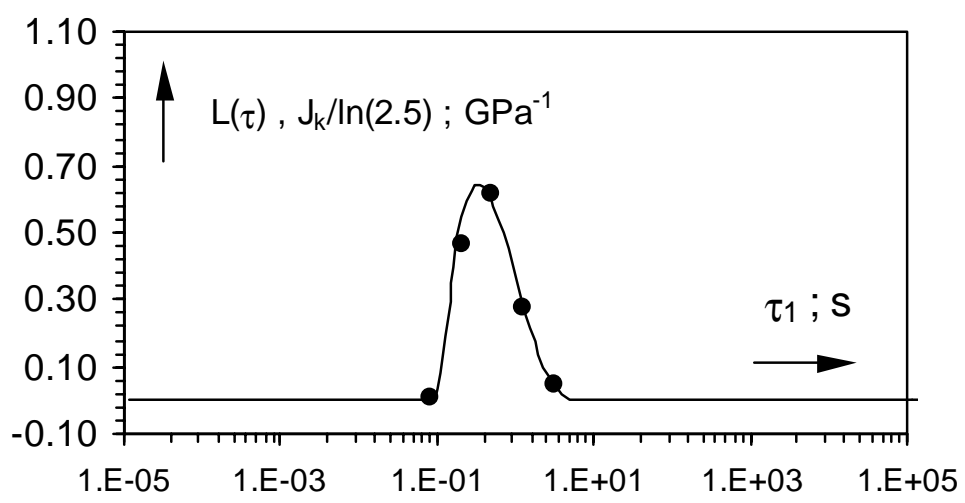

Figure 2: Normalised retardation components $J k / \ln (a)$ for $a=2.5$ vs exact retardation spectrum, Eq. 20. 


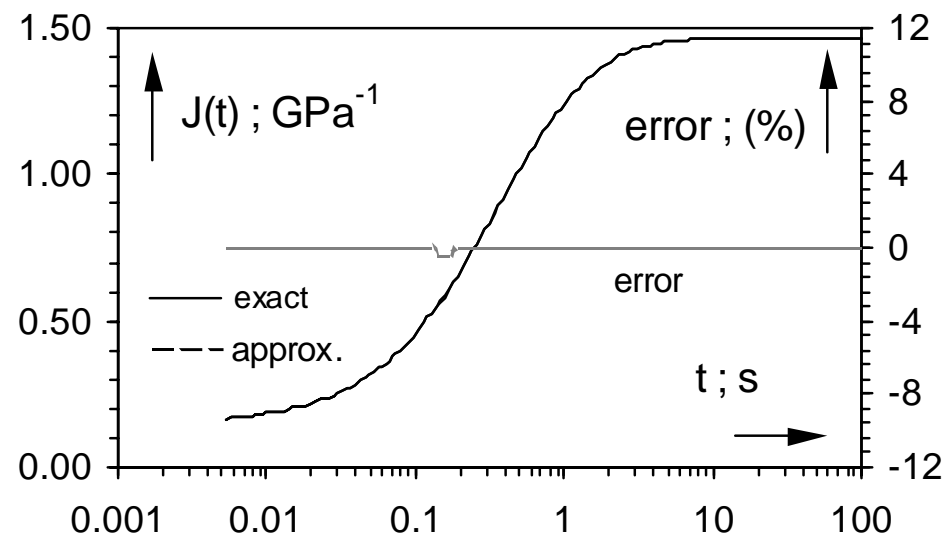

Figure 3: Approximated creep compliance for $a=2.5$ vs exact creep compliance as calculated from spectrum given by Eq. 20.

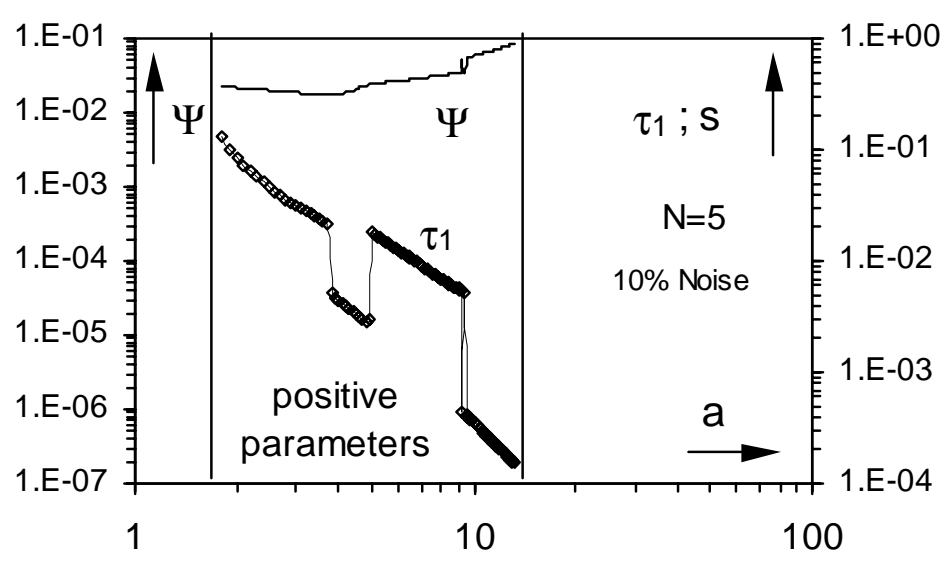

Figure 4: Minimum of $\Psi$ and the corespondent retardation time, $\tau_{1}$, for the calculation of the dynamic compliances with $\pm 10 \%$ noise, as functions of the logarithmic distance, $a$, between the retardation times. 


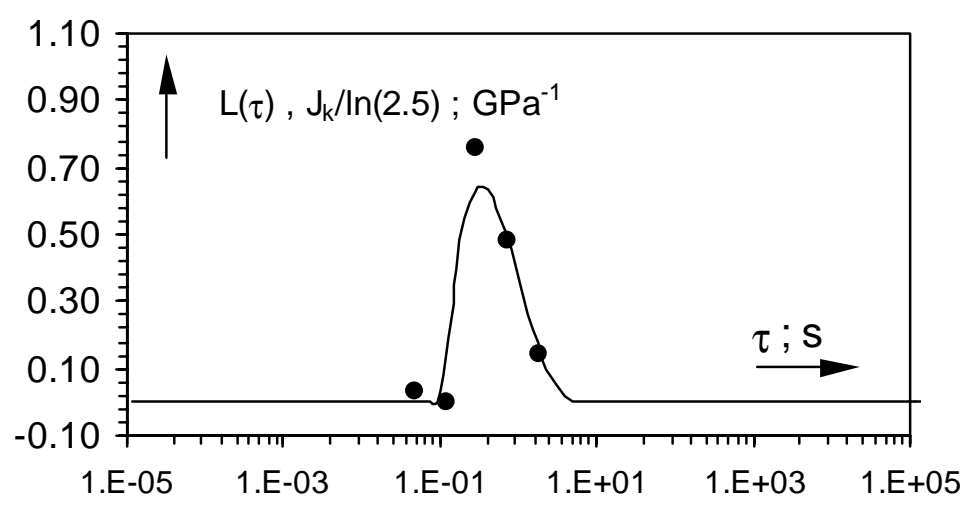

Figure 5: Normalised retardation components $J k / \ln (a)$ for $a=2.5$ and for $\pm 10 \%$ noise dynamic data $(\partial=0.1)$ vs exact retardation spectrum, Eq. 20.

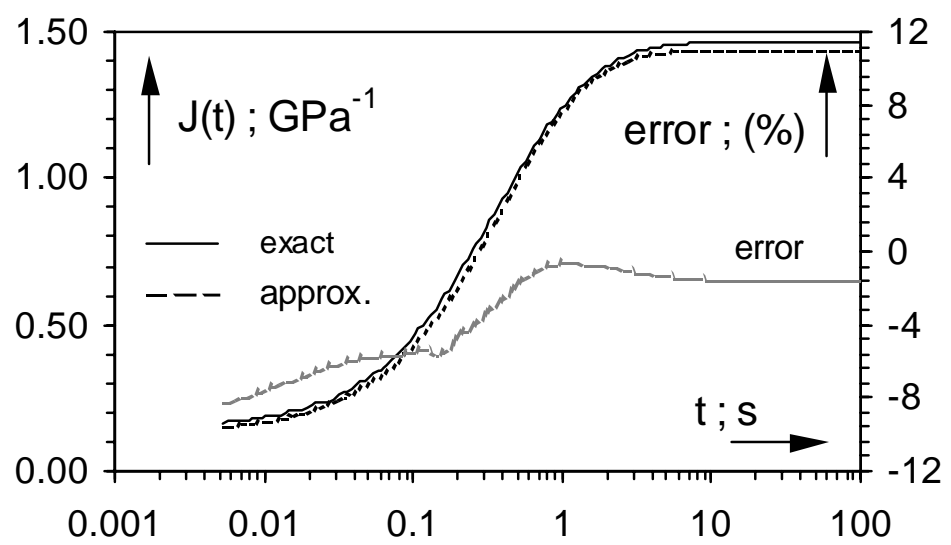

Figure 6: Approximated creep compliance for $a=2.5$ and for $\pm 10 \%$ noise dynamic data $(\partial=0.1)$ vs exact creep compliance as calculated from spectrum given by Eq. 20. 


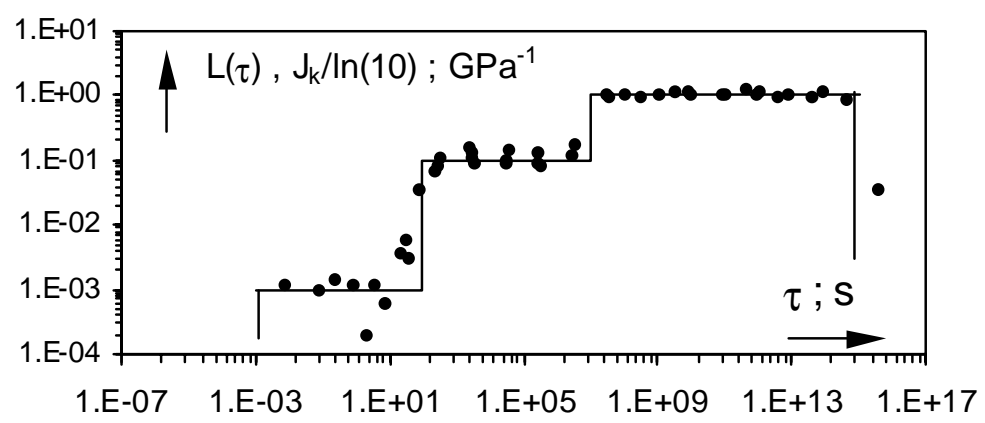

Figure 7: Double-logarithmic plot of the normalised retardation components $J k / \ln (a)$ for $a=10$ vs the exact retardation spectrum, Eq. 21.

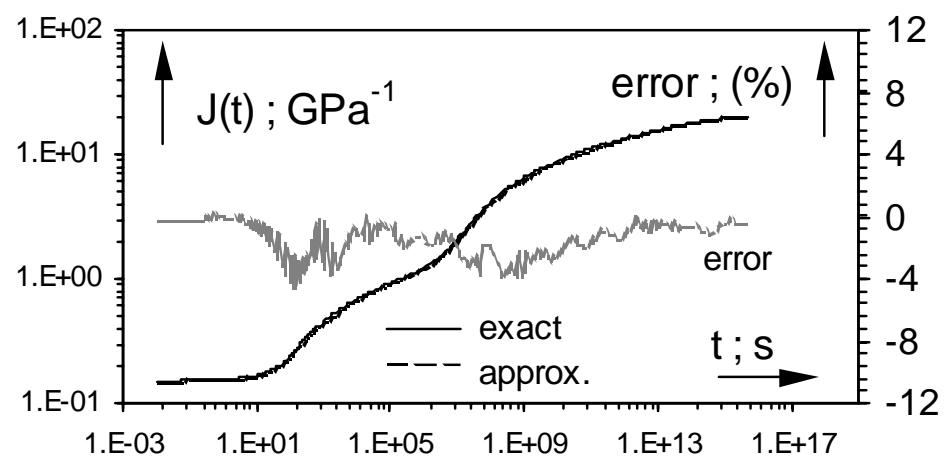

Figure 8: Double-logarithmic plot of the approximated creep compliance for $a=10$ vs exact creep compliance as calculated from spectrum given by Eq. 21. 


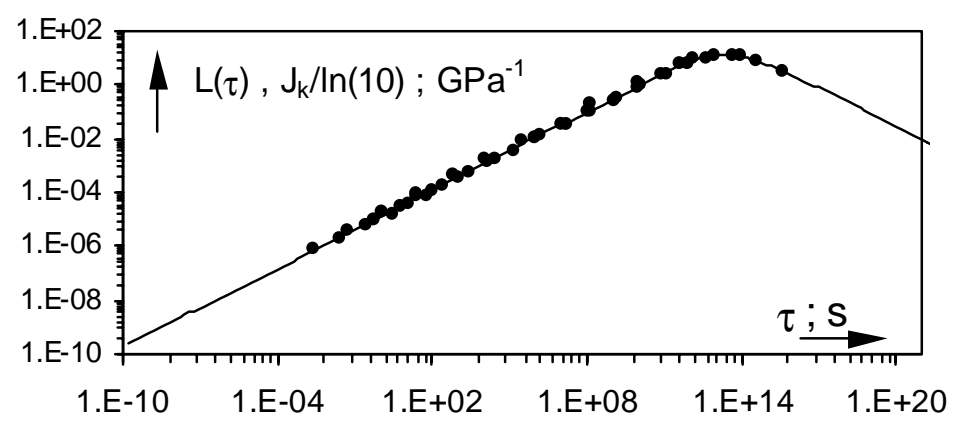

Figure 9: Double-logarithmic plot of the normalised retardation components $J k / \ln (a)$ for $a=10$ vs exact retardation spectrum, Eq. 22.

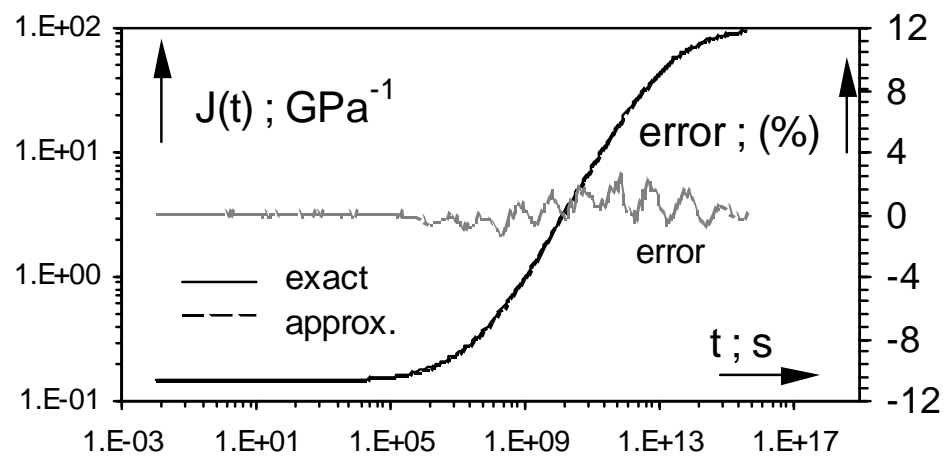

Figure 10: Double-logarithmic plot of the approximated creep compliance for $a=10$ vs exact creep compliance as calculated from spectrum given by Eq. 22 


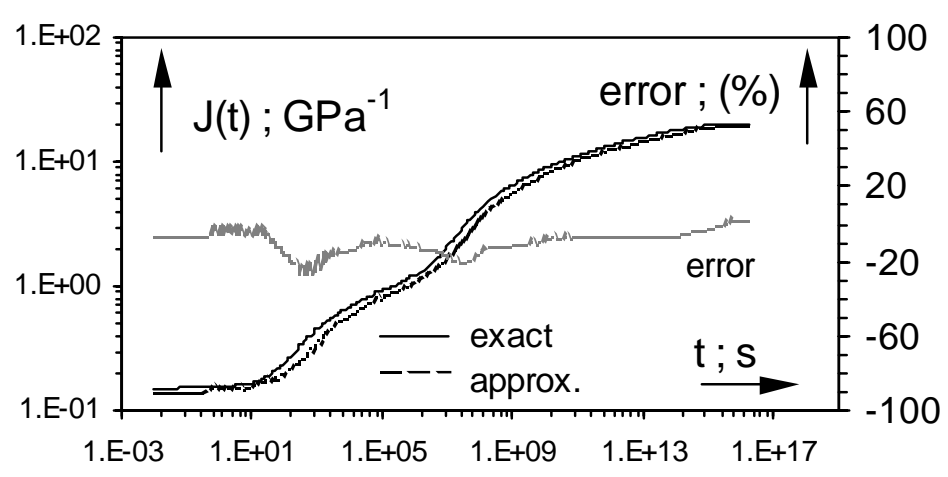

Figure 11: Double-logarithmic plot of the approximated creep compliance for $a=10$ and for $\pm 10 \%$ noise dynamic data $(\partial=0.1)$ vs exact creep compliance as calculated from the spectrum given by Eq. 21 .

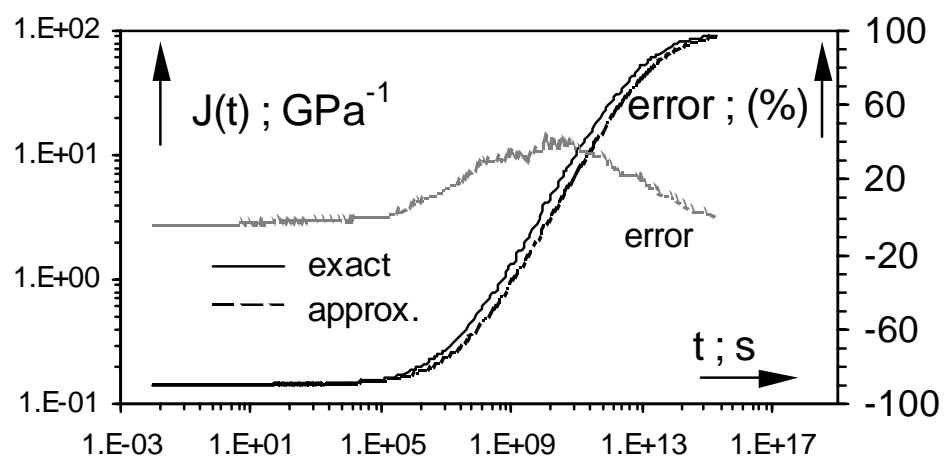

Figure 12: Double-logarithmic plot of the approximated creep compliance for $a=10$ and for $\pm 10 \%$ noise dynamic data $(\partial=0.1)$ vs exact creep compliance as calculated from the spectrum given by Eq. 22 . 